\title{
UNIQUENESS OF THE SRB MEASURE FOR PIECEWISE EXPANDING WEAKLY COUPLED MAP LATTICES IN ANY DIMENSION
}

\author{
GERHARD KELLER AND CARLANGELO LIVERANI
}

\begin{abstract}
We prove the existence of a unique SRB measure for a wide range of multidimensional weakly coupled map lattices. These include piecewise expanding maps with diffusive coupling.
\end{abstract}

\section{INTRODUCTION}

The field of expanding coupled map lattices has witnessed an impressive series of results since the late 1980's. Starting with [7] numerous authors contributed to the exploration of ergodic and statistical properties of invariant measures for such systems, see e.g. 1, 2, 3, 4, 5, 6, 8, 9, 10, 11, 12, 13, 14, 15, 16, 17, 18, 19, 20, 29, 31, 32, 34 35. In all these publications the single site maps are hyperbolic or expanding (local) diffeomorphisms of a smooth manifold, and the coupling is modeled by a "diffeomorphism" of the infinite-dimensional state space. Only a few publications used a different approach which allows to treat also piecewise expanding maps and such a common coupling like the diffusive nearest neighbour coupling [21,22, 23, 24 , [27, $28,25 \mid 26,33$.

Yet, the state of the field it is still far from satisfactory. One of the outstanding open problems is to substantiate rigorously the numerical picture of a phase transition given in [30]. The model considered in the aforementioned paper is a $\mathbb{Z}^{2}$ lattice of expanding Lasota-Yorke like maps, coupled by a diffusive nearest neighbour interaction. As the coupling parameter increases from zero the authors notice the transition from a situation in which only one invariant measure describes the statistical properties of the system to one in which two relevant invariant measures appear (a phase transition, indeed). After more than ten years no aspect of such a picture has been rigorously proven. In the present paper we prove the first (easier) part of the picture: the existence of only one "relevant" (that is SRB) measure for small coupling.

The proof is surprisingly elementary. It combines the following key ideas:

(i) The starting point is a Lasota-Yorke type inequality for coupled systems (cf. 24, 28,).

(ii) The transfer operator of the uncoupled system is interpreted as a tensor product operator of the single site transfer operators (cf. 33]). This allows to make optimal use of the strong mixing properties of the single site systems.

Date: November 5, 2004.

1991 Mathematics Subject Classification. 37C30,37L60,37D20.

Key words and phrases. Coupled map lattice, SRB measure, exponential decay of correlations.

The essential part of this research was done during an ESF explorative workshop at the MaxPlanck-Institute for Mathamatics, Bonn. We thank both institutions for their support. 
(iii) A "site-by-site" decoupling procedure allows to reduce the dynamics of the coupled system "locally" to dynamics of tensor-product type at the cost of only small errors (cf. [25, 26]).

(iv) The aforementioned small errors are not controlled in the original system but in a huge extension of that system. This is the essential new idea of this paper. We believe that it has applications far beyond the present model; indeed, we expect it to be useful for all kinds of weakly coupled systems where the local dynamics can be described by linear operators with an isolated simple leading eigenvalue. Typical examples are high temperature stochastic Ising models or weakly coupled uniformly contractive iterated function systems.

The plan of the paper is as follows: Section 2 details the model and describes the basic result obtained in the paper. Section 3 describes the already mentioned extension of the system and how to use it to get the main estimate of the paper. Secion 4 contains the proof of the main theorem based on the results of Section 3 . Finally, Section 5 contains the proof for the case of more general coupling, but with an extra simplifying assumption on the single site map.

\section{The MODEL AND THE RESUlT}

Given a compact interval $I \subset \mathbb{R}$ we will consider the phase space $\Omega:=I^{\Lambda}$, where either $\Lambda=\mathbb{Z}^{d}$ or $\Lambda$ is a box in $\mathbb{Z}^{d} \mathbb{1}$ In the following we always assume $I=[0,1]$ and $\boldsymbol{O}=(0, \ldots, 0) \in \Lambda$, as this can be done without loss of generality

We will have a single site dynamics given by the map $\tau: I \rightarrow I$. We assume $\tau$ to be a piecewise $\mathcal{C}^{2}$ map from $I$ to $I$ with singularities at $\zeta_{1}, \ldots, \zeta_{N-1} \in(0,1)$ in the sense that $\tau$ is monotone and $\mathcal{C}^{2}$ on each component of $I \backslash\left\{\zeta_{0}=0, \zeta_{1}, \ldots, \zeta_{N-1}, \zeta_{N}=\right.$ $1\}$. We assume that $\tau^{\prime \prime} /\left(\tau^{\prime}\right)^{2}$ is bounded and that inf $\left|\tau^{\prime}\right|>23$

Next, we define the unperturbed dynamics $T_{0}: \Omega \rightarrow \Omega$ by $\left[T_{0}(x)\right]_{p}:=\tau\left(x_{p}\right)$.

To define the perturbed dynamics we introduce couplings $\Phi_{\epsilon}: \Omega \rightarrow \Omega$ of the form $\Phi_{\epsilon}(\boldsymbol{x}):=\boldsymbol{x}+A_{\epsilon}(\boldsymbol{x})$. We call $\Phi_{\epsilon}$ a $\left(a_{1}, a_{2}\right)$-coupling, if there are operators $A^{\prime}, A^{\prime \prime}: \ell^{1}(\Lambda) \rightarrow \ell^{1}(\Lambda)$ with $a_{1}=\left\|A^{\prime}\right\|_{1}, a_{2}=\left\|A^{\prime \prime}\right\|_{1}$ (maximal column sum norm) such that for all $\boldsymbol{k}, \boldsymbol{p}, \boldsymbol{q} \in \Lambda$

$$
\left|\left(A_{\epsilon}\right)_{p}\right| \leq 2|\epsilon|, \quad\left|\left(D A_{\epsilon}\right)_{q p}\right| \leq 2|\epsilon| A^{\prime}{ }_{q p}, \quad\left|\partial_{k}\left(D A_{\epsilon}\right)_{q p}\right| \leq 2|\epsilon| A^{\prime \prime}{ }_{q p} .
$$

Here $\partial_{k}$ denotes the partial derivative with respect to $x_{k}$. In addition, we say that $\Phi_{\epsilon}$ has finite coupling range $w>0$, if $\partial_{p} \Phi_{\epsilon, \boldsymbol{q}}=0$ whenever $|\boldsymbol{p}-\boldsymbol{q}|>\boldsymbol{w}$. So $A^{\prime}{ }_{q p}=A^{\prime \prime}{ }_{q p}=0$ when $|\boldsymbol{p}-\boldsymbol{q}|>w$. We say that a coupling has short range if it is not of finite range and there exist constants $L>0$ and $\gamma \in(0,1)$ such that $A^{\prime}{ }_{q p}+A^{\prime \prime}{ }_{q p} \leq L \gamma^{|p-q|}$. Similarly, we say that a coupling has long range if it is neither finite range nor short range and there exists $c^{\prime}>0$ such that $A^{\prime}{ }_{q p}+A^{\prime \prime}{ }_{q p} \leq L|\boldsymbol{p}-\boldsymbol{q}|^{c^{\prime}}$. The diffusive nearest neighbor coupling used in [30, and in much of the numerical

\footnotetext{
${ }^{1} \mathrm{By}$ box here, and in the following, we mean a hypercube. Of course much more general shapes can be considered by the same arguments, yet for shapes with too large a boundary problems may arise. To avoid all the related technicalities we confine ourselves to the above mentioned case.

${ }^{2}$ The reader should be aware that there is nothing special about $\mathbb{Z}^{d}$, any other lattice (or graph) can be treated similarly, provided the number of different sites that can be reached from a given site along a path of length $n$ grows at most subexponentially in $n$.

${ }^{3}$ Under mild additional assumptions on $\tau$ also maps with $1<\inf \left|\tau^{\prime}\right| \leq 2$ can be treated. The complications, which arise in the proof of a Lasota-Yorke type inequality, were overcome in 28, see also the discussions of this point in 22 and in 26 Footnote 14].
} 
literature, is defined by

$$
\left[\Phi_{\epsilon}(\boldsymbol{x})\right]_{p}=x_{p}+\frac{\epsilon}{2 d} \sum_{|\boldsymbol{p}-\boldsymbol{q}|=1}\left(x_{q}-x_{\boldsymbol{p}}\right) \quad(\boldsymbol{p} \in \Lambda),
$$

and it is an example of a $(1,0)$-coupling with range $w=14$

The dynamics $T_{\epsilon}: \Omega \rightarrow \Omega$ that we wish to investigate is then defined as

$$
T_{\epsilon}:=\Phi_{\epsilon} \circ T_{0},
$$

and, more precisely, we wish to investigate its invariant measures in some appropriate class. Let $\mathcal{M}(\Omega)$ be the set of signed Borel measures on $\Omega 5$ To state the main result of the paper we need to introduce the concept of measures of bounded variation. Let $\mathcal{I}$ be the set of all boxes $\Lambda_{1} \subset \Lambda$. For each $\Lambda_{1} \in \mathcal{I}$ we define 6

$$
\begin{aligned}
\operatorname{Var} \mu & :=\sup _{p \in \Lambda|\varphi|_{\mathcal{C}^{0}(\Omega)} \leq 1} \sup _{\operatorname{Var}_{\Lambda_{1}} \mu}:=\sup _{p \in \Lambda_{1}} \sup _{|\varphi|_{\mathcal{C}^{0}\left(I^{\left.\Lambda_{1}\right)} \leq 1\right.}} \mu\left(\partial_{p} \varphi\right) .
\end{aligned}
$$

It is easy to prove that the set $\mathcal{B}(\Omega):=\{\mu \in \mathcal{M}(\Omega)$ : $\operatorname{Var} \mu<\infty\}$ consists of measures whose finite dimensional marginals are absolutely continuous with respect to Lebesgue and the density is a function of bounded variation [25]. In addition, such measures have finite entropy density with respect to Lebesgue [26] Corollary 5]. In fact, "Var" is a norm and, with this norm, $\mathcal{B}(\Omega)$ is a Banach space

It is also useful to introduce the usual total variation norm on signed measures:

$$
|\mu|:=\sup _{|\varphi|_{\mathcal{C}^{0}(\Omega)} \leq 1} \mu(\varphi) .
$$

Just like in [25, Sect. 3.3] one checks easily that

$$
|\mu| \leq \frac{1}{2} \operatorname{Var} \mu .
$$

As we are interested in studying observable invariant measures, we must restrict to a subclass of the class of all measures in order to make relevant statements. Clearly, $\mathcal{M}(\Omega)$ is too large for our purposes, but on the other hand, in the case in which $\Lambda$ is infinite, $\mathcal{B}(\Omega)$ is quite small. As usual in thermodynamics, it makes sense to require some condition on the growth of the relevant quantity with respect to the volume. Let $\mathcal{M}_{v}(\Omega)$ be the closure of the set

$$
\left\{\mu \in \mathcal{M}(\Omega): \forall \eta>0 \sup _{\Lambda_{1} \in \mathcal{I}} e^{-\eta\left|\Lambda_{1}\right|^{\frac{1}{d}}} \operatorname{Var}_{\Lambda_{1}} \mu<\infty\right\},
$$

with respect to the norm $|\cdot|$. Clearly, $\mathcal{M}_{v}(\Omega)$ consists of measures that can be uniformly approximated by measures with absolutely continuous finite dimensional marginals whose densities are functions of bounded variation with the variation growing less than exponentially in the size of the boxes.

\footnotetext{
${ }^{4}$ Of course, if $\Lambda \neq \mathbb{Z}^{d}$, then the sum in 2.2 can involve sites not in $\Lambda$. To properly define the dynamics it is then necessary to supply some boundary conditions, that is to specify some fixed value for $x_{q}, q \notin \Lambda$.

${ }^{5}$ The topology that we use on $\Omega$ is the product one.

${ }^{6}$ Here, and in the following, we will consider $\mathcal{C}^{0}\left(I^{\Lambda_{1}}\right)$ as a subspace of $\mathcal{C}^{0}(\Omega)$ by the obvious inclusion. Also the sup is restricted to functions derivable with respect to $x_{p}$.

${ }^{7}$ See 26 for a careful discussion of bounded variation in the present context and the relevant associated properties.
} 
The results of this paper can be summarized, a bit loosely, as follow.

Theorem 2.1. For each $\left(a_{1}, a_{2}\right)$-coupling of finite range $w$, there exists $\epsilon_{0}>0$ such that, for each $|\epsilon|<\epsilon_{0}$, the dynamical system $\left(\Omega, T_{\epsilon}\right)$ has a unique invariant measure $\mu_{\epsilon}$ in $\mathcal{M}_{v}(\Omega)$ 专 In addition, $\mu_{\epsilon}$ belongs to $\mathcal{B}(\Omega)$, is exponentially mixing both in time and in space, and it is the SRB measure of the system.

The proof, which also makes precise the statement, can be found in Section 4

To obtain such a result we consider the dynamics acting directly on the measures via the linear operator $T_{\epsilon}^{*} \mu(A):=\mu\left(T_{\epsilon}^{-1} A\right)$ (for each measurable set $A$ ). The basic facts concerning the operator $T_{\epsilon}^{*}$ are detailed in the following lemma.

Lemma 2.2 (Lasota-Yorke inequality). For each $\left(a_{1}, a_{2}\right)$-coupling, there exist $\epsilon_{1}>$ $0, \lambda>1$, and $a, b>0$ such that, for each $|\epsilon|<\epsilon_{1}$, the operator $T_{\epsilon}^{*}$ is well defined as an operator on $\mathcal{B}(\Omega)$. In addition, for each $\mu \in \mathcal{B}(\Omega)$ holds true

$$
\begin{aligned}
\left|T_{\epsilon}^{*} \mu\right| & \leq|\mu| \\
\operatorname{Var}\left(T_{\epsilon}^{* n} \mu\right) & \leq a \lambda^{-n} \operatorname{Var} \mu+b|\mu| .
\end{aligned}
$$

This is the special case $\theta=1$ of Proposition 4 in [26] (see below for the meaning of $\theta$ ). Observe that the proof given there for $\Lambda=\mathbb{Z}$ applies (only if $\theta=1$ !) without changes to $\Lambda \subseteq \mathbb{Z}^{d}$.

From preceding experience it is also useful to consider larger Banach spaces: first define, for each $\theta \in(0,1]$, a norm

$$
\|\mu\|_{\theta}:=\sup _{\Lambda_{1} \in \mathcal{I}} \theta^{\left|\Lambda_{1}\right|} \operatorname{Var}_{\Lambda_{1}} \mu
$$

on $\mathcal{B}(\Omega)$. Then we let $\mathcal{B}(\Omega, \theta)$ be the completion of $\mathcal{B}(\Omega)$ with respect to this norm $\mathbb{G}$ Observe that $\|\mu\|_{\theta=1}=\operatorname{Var} \mu$.

The key estimate on which Theorem 2.1 relies is given in the following lemma whose proof is the content of the next section. Let

$$
\mathcal{B}^{0}(\Omega):=\{\mu \in \mathcal{B}(\Omega): \mu(1)=0\} .
$$

Lemma 2.3. Recall that $\Omega=I^{\Lambda}$. For each $\left(a_{1}, a_{2}\right)$-coupling with finite range $w$, there exist $\sigma \in(0,1)$ and $C, \epsilon_{2}>0$ such that, for all $|\epsilon|<\epsilon_{2}, \mu \in \mathcal{B}^{0}(\Omega), \theta \in(0,1)$, and $n \in \mathbb{N}$ holds true

$$
\left\|T_{\epsilon}^{* n} \mu\right\|_{\theta} \leq C \sigma^{n} \min \left\{|\Lambda|,|e \ln \theta|^{-1}\right\} \operatorname{Var} \mu .
$$

Finally, we wish to emphasize the power of the approach by showing the possibility of extending it to more more general settings. Short range interactions can be treated in a spirit similar to the one used for the finite range. Nevertheless, the technical construction becomes inevitably more involved. For the long range case the situation looks still similar but one cannot expect an exponential convergence to the invariant measure, so one cannot simply rely on an estimate of the spectral radius of the covering dynamics and the story is bound to acquire an extra layer of complexity.

\footnotetext{
${ }^{8}$ If the coupling is defined only for nonnegative $\epsilon$ (as it is the case for the diffusive nearest neighbour coupling on $\Omega$ ), this has to be understood as "for each $\epsilon \in\left[0, \epsilon_{0}\right) \ldots$ " here and in the sequel.

${ }^{9}$ Note that if $|\Lambda|=\infty$ and $0<\theta<1$, then $\mathcal{B}(\Omega, \theta)$ contains objects that are not signed measures, see 2526$]$ for details.
} 
To keep the technicalities to a minimum here we content ourself with the following result (proved in Section (5) concerning the short range case with an additional assumption on the single site map.

Theorem 2.4. If the map $\tau$ is Lipschitz, then for each $\left(a_{1}, a_{2}\right)$-coupling of short range, there exists $\epsilon_{0}>0$ such that, for each $|\epsilon|<\epsilon_{0}$, the dynamical system $\left(\Omega, T_{\epsilon}\right)$ has a unique invariant measure $\mu_{\epsilon}$ in $\mathcal{M}_{v}(\Omega)$. In addition, $\mu_{\epsilon}$ belongs to $\mathcal{B}(\Omega)$, is exponentially mixing both in time and in space, and it is the SRB measure of the system.

\section{Lifting the system, Proof of Lemma 2.3}

From now on we will suppress the dependence on $\Omega$ in notations like $\mathcal{B}(\Omega)$.

The basic idea of the present work is to define an extension of the linear system $\left(T_{\epsilon}^{*}, \mathcal{B}\right)$ and to study its spectral properties instead of the ones of $T_{\epsilon}^{*}$. To do so define

$$
\mathcal{B}_{p}:=\left\{\mu \in \mathcal{B}: \partial_{p} \varphi=0 \Rightarrow \mu(\varphi)=0\right\} .
$$

Remark that $\mathcal{B}_{p} \subset \mathcal{B}^{0}$. We can then define $\overline{\mathcal{B}}_{\Lambda}:=\mathrm{X}_{p \in \Lambda} \mathcal{B}_{p}$ and $\overline{\mathcal{B}}^{0}:=\left(\mathcal{B}^{0}\right)^{\Lambda}$, these are Banach spaces with the norm $\|\bar{\mu}\|:=\sup _{p \in \Lambda} \operatorname{Var} \mu_{p}$.

As $T_{\epsilon}^{*}\left(\mathcal{B}^{0}\right) \subseteq \mathcal{B}^{0}$, the (coupled) dynamics is easily lifted to $\overline{\mathcal{B}}^{0}$, namely $\bar{T}_{\epsilon}: \overline{\mathcal{B}}^{0} \rightarrow$ $\overline{\mathcal{B}}^{0}$ can be defined as $\left(\bar{T}_{\epsilon} \bar{\mu}\right)_{p}:=T_{\epsilon}^{*} \mu_{p}$. However, only in the uncoupled case $\epsilon=0$ the operator $\bar{T}_{0}$ leaves the subspace $\overline{\mathcal{B}}_{\Lambda}$ of $\overline{\mathcal{B}}^{0}$ invariant. Since the invariance of this subspace - also under a suitable lift of $T_{\epsilon}^{*}$ when $\epsilon \neq 0$ - is crucial for our approach, we need to proceed more carefully in choosing a suitable lift.

To start with, let us consider some total ordering $\sigma: \mathbb{N} \rightarrow \mathbb{Z}^{d}$ of $\mathbb{Z}^{d}$ with the property 10

$$
c^{-1} i^{\frac{1}{d}} \leq|\sigma(i)| \leq c i^{\frac{1}{d}} .
$$

For each $\boldsymbol{p}=\boldsymbol{p}+\sigma(0)$ and $\boldsymbol{q}=\boldsymbol{p}+\sigma(i)$ in $\mathbb{Z}^{d}$ one can then define the (partial) telescoping operators $\Pi_{p, q}$ acting on test functions 11

$$
\Pi_{p, q} \varphi(\boldsymbol{x}):=\int \varphi(\boldsymbol{x}) d x_{\boldsymbol{p}} \cdots d x_{p+\sigma(i-1)}-\int \varphi(\boldsymbol{x}) d x_{p} \cdots d x_{p+\sigma(i)} .
$$

Essentially $\boldsymbol{p} \in \Lambda$ specifies the point from which one starts to telescope and $\boldsymbol{q}$ how far one is in the telescoping procedure. Note that $\Pi_{p, q} \varphi=0$ if $\partial_{q} \varphi=0$, and that $\Pi_{p, q} \varphi$ does not depend on the variables contained inside a box of size $c^{-2}|\boldsymbol{q}-\boldsymbol{p}|$ centered at $p$ We then define the lift $\Psi: \mathcal{B}^{0} \rightarrow \overline{\mathcal{B}}_{\Lambda}$ by

$$
\Psi(\mu)_{q}:=\Pi_{\mathbf{0}, q}^{*} \mu \text {. }
$$

and the projection map $P: \overline{\mathcal{B}}_{\Lambda} \rightarrow \mathcal{B}(\theta)$ by

$$
P(\bar{\mu}):=\sum_{p \in \Lambda} \mu_{p}
$$

\footnotetext{
${ }^{10}$ For example, on a square lattice one can spiral out from zero on larger and larger squares.

${ }^{11}$ Here $\varphi \in \mathcal{C}^{0}\left(I^{\mathbb{Z}^{d}}\right)$. This definition suffices in view of the identification already mentioned in Footnote 6

${ }^{12}$ Indeed, if $\boldsymbol{q}^{\prime} \in \Lambda,\left|\boldsymbol{q}^{\prime}-\boldsymbol{p}\right|<c^{-2}|\boldsymbol{q}-\boldsymbol{p}|$, then, by [3.1], $\sigma^{-1}\left(\boldsymbol{q}^{\prime}-\boldsymbol{p}\right) \leq c^{d}\left|\boldsymbol{q}^{\prime}-\boldsymbol{p}\right|^{d}<c^{-d}|\boldsymbol{q}-\boldsymbol{p}|^{d} \leq$ $\sigma^{-1}(\boldsymbol{q}-\boldsymbol{p})$. Hence $\boldsymbol{q}^{\prime}$ has already been integrated out in $\Pi_{p, q}$.
} 
which is well defined for $\theta \in(0,1)$ even if $\Lambda$ is infinite 13 In fact,

$$
\|P\|_{\overline{\mathcal{B}}_{\Lambda} \rightarrow \mathcal{B}(\theta)} \leq \min \left\{|\Lambda|,|e \ln \theta|^{-1}\right\},
$$

because $\operatorname{Var}_{\Lambda_{1}} \mu_{p}=0$ if $p \in \Lambda \backslash \Lambda_{1}$. Observe also that, for each function $\varphi$ depending only on finitely many variables and for each $\mu \in \mathcal{B}^{0}$,

$$
P(\Psi(\mu))(\varphi)=\mu(\varphi) .
$$

In addition, it is easy to verify that

$$
P \bar{T}_{\epsilon}=T_{\epsilon}^{*} P .
$$

As remarked before, since $\bar{T}_{\epsilon} \overline{\mathcal{B}}_{\Lambda} \not \subset \overline{\mathcal{B}}_{\Lambda}$, we need some way to go back to the space $\overline{\mathcal{B}}_{\Lambda}$. This is achieved via the (partially defined!) telescoping operator $\bar{H}: \overline{\mathcal{B}}^{0} \rightarrow \overline{\mathcal{B}}_{\Lambda}$,

$$
(\bar{H} \mu)_{q}=\sum_{p \in \Lambda} \Pi_{p, q}^{*} \mu_{p} .
$$

Indeed, the infinite sum is not always well defined, but as we only consider finite range couplings, the operators

$$
\bar{T}_{\epsilon, m}:=\bar{H} \bar{T}_{\epsilon}^{m}(m \geq 1)
$$

are always well defined on $\overline{\mathcal{B}}_{\Lambda}$, as the next lemma shows.

Lemma 3.1. Let $m \geq 1$. The linear operator $\bar{T}_{\epsilon, m}: \overline{\mathcal{B}}_{\Lambda} \rightarrow \overline{\mathcal{B}}_{\Lambda}$ is well defined, and

$$
\left\|\bar{T}_{\epsilon, m} \bar{\mu}\right\| \leq C(w m)^{d} \sup _{p \in \Lambda} \operatorname{Var}\left(T_{\epsilon}^{* m} \mu_{\boldsymbol{p}}\right) .
$$

Proof. Let $\varphi \in \mathcal{C}^{1}(\Omega)$. As noted above, $\partial_{q^{\prime}}\left(\Pi_{p, q} \varphi\right)=0$ for all $q^{\prime} \in \Lambda$ such that $\left|\boldsymbol{q}^{\prime}-\boldsymbol{p}\right|<c^{-2}|\boldsymbol{q}-\boldsymbol{p}|$. Consequently, $\partial_{\boldsymbol{p}}\left(\left(\Pi_{\boldsymbol{p}, q} \varphi\right) \circ T_{\epsilon}^{m}\right)=0$ provided $|\boldsymbol{q}-\boldsymbol{p}|>c^{2} m w$. Therefore, for $\mu_{p} \in \mathcal{B}_{p}$,

$$
\left(\Pi_{\boldsymbol{p}, q}^{*} T_{\epsilon}^{* m} \mu_{\boldsymbol{p}}\right)(\varphi)=\mu_{\boldsymbol{p}}\left(\left(\Pi_{p, q} \varphi\right) \circ T_{\epsilon}^{m}\right)=0 \quad \text { if }|\boldsymbol{q}-\boldsymbol{p}|>c^{2} m w .
$$

It follows that for $\bar{\mu} \in \overline{\mathcal{B}}_{\Lambda}, \bar{\mu}=\left(\mu_{p}\right)_{p \in \Lambda}$,

$$
\left(\bar{T}_{\epsilon, m} \bar{\mu}\right)_{q}=\left(\bar{H} \bar{T}_{\epsilon}^{m} \bar{\mu}\right)_{q}=\sum_{|q-p| \leq c^{2} m w} \Pi_{p, q}^{*} T_{\epsilon}^{* m} \mu_{p}
$$

is well defined, and

$$
\left\|\bar{T}_{\epsilon, m} \bar{\mu}\right\|=\sup _{q \in \Lambda} \operatorname{Var}\left(\bar{H} \bar{T}_{\epsilon}^{m} \bar{\mu}\right)_{q} \leq 2\left(2 c^{2} m w\right)^{d} \sup _{p \in \Lambda} \operatorname{Var}\left(T_{\epsilon}^{* m} \mu_{p}\right) .
$$

Recall from (3.3) that $P \bar{T}_{\epsilon}=T_{\epsilon}^{*} P$. As $P \bar{H}=P$ whenever these operators are well defined, it follows

$$
P \bar{T}_{\epsilon, m}=T_{\epsilon}^{* m} P .
$$

So we can use $\bar{T}_{\epsilon, m}: \overline{\mathcal{B}}_{\Lambda} \rightarrow \overline{\mathcal{B}}_{\Lambda}$ as a covering dynamics for $T_{\epsilon}^{* m}: \mathcal{B}^{0} \rightarrow \mathcal{B}^{0}$.

\footnotetext{
${ }^{13}$ Note that, on each local test function, the sum reduces to a finite sum.
} 
In particular, for each $n \geq 1$, we have the commuting diagram

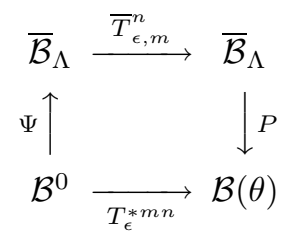

which makes sense since, by Lemma $3.1 \bar{T}_{\epsilon, m}$ is a bounded operator on $\overline{\mathcal{B}}_{\Lambda}$.

To conclude we need to have a closer look at the operator $\bar{T}_{\epsilon, m}$. Recall that the sum in (3.7) is a finite sum as the interaction has finite range.

Next, writing $m=m_{1}+m_{2}$, for each $\bar{\mu} \in \overline{\mathcal{B}}_{\Lambda}$ and each $\boldsymbol{p} \in \Lambda$, holds

$$
\operatorname{Var}\left(T_{\epsilon}^{* m} \mu_{\boldsymbol{p}}\right) \leq a^{\prime} \lambda^{-m_{1}} \operatorname{Var} \mu_{p}+b\left|T_{\epsilon}^{* m_{2}} \mu_{\boldsymbol{p}}\right|
$$

with $a^{\prime}=a\left(a+\frac{1}{2} b\right)$, see Lemma 2.2 and eq. (2.5). In order to profit from the strong mixing properties of the single site operator we use a decoupling trick originally introduced in a similar context in [3]: approximate $\Phi_{\epsilon}$ by $\Phi_{\epsilon}^{p}$, where site $\boldsymbol{p}$ is decoupled from all other sites. To this end we introduce the following notation: let $\bar{\iota}_{p}: I^{\Lambda} \rightarrow I^{\Lambda}$ be the map $\left(\bar{\iota}_{\boldsymbol{p}}(\boldsymbol{x})\right)_{\boldsymbol{q}}=x_{\boldsymbol{q}}$ if $\boldsymbol{q} \neq \boldsymbol{p}$ and $\left(\bar{\iota}_{\boldsymbol{p}}(\boldsymbol{x})\right)_{\boldsymbol{p}}=0$. Then define $\Phi_{\epsilon}^{p}: I^{\Lambda} \rightarrow I^{\Lambda}$,

$$
\left(\Phi_{\epsilon}^{p}(\boldsymbol{x})\right)_{q}= \begin{cases}x_{\boldsymbol{p}} & \text { if } \boldsymbol{q}=\boldsymbol{p} \\ \left(\Phi_{\epsilon}\left(\bar{\iota}_{\boldsymbol{p}}(\boldsymbol{x})\right)_{\boldsymbol{q}}\right. & \text { if } \boldsymbol{q} \neq \boldsymbol{p}\end{cases}
$$

Note that

$$
\left(D \Phi_{\epsilon}^{p}\right)_{q p}=\delta_{q p}
$$

This implies

$$
\left(\Phi_{\epsilon}^{p}\right)^{*}\left(\mathcal{B}_{p}\right) \subseteq \mathcal{B}_{p}
$$

It is then natural to define the decoupled dynamics $T_{\epsilon, p}:=\Phi_{\epsilon}^{p} \circ T_{0}$. Observe that $\left(T_{\epsilon, p}^{m} x\right)_{p}=\tau^{m}\left(x_{p}\right)$.

Here is a basic estimate for comparing different couplings. It is a variant of [26, Proposition 5], and we give its proof in the appendix.

Lemma 3.2. The lemma consists of two parts:

a) Let $F, \tilde{F}: \Omega \rightarrow \Omega$ be two Lipschitz map 14 with Lipschitz constant $L>0$ that are close in the following sense: There are constants $K_{0}, K_{1}, K_{2}>0$ such that

(i) $\sum_{q \in \Lambda} \sup _{\boldsymbol{x}}\left|\tilde{F}_{\boldsymbol{q}}(\boldsymbol{x})-F_{\boldsymbol{q}}(\boldsymbol{x})\right| \leq K_{0}$,

(ii) $\sum_{\boldsymbol{q} \in \Lambda} \sup _{\boldsymbol{p} \in \Lambda} \sup _{\boldsymbol{x}_{\neq p}} \int_{I}\left|\partial_{\boldsymbol{p}} \tilde{F}_{\boldsymbol{q}}\left(\boldsymbol{x}_{\neq \boldsymbol{p}}, \xi\right)-\partial_{\boldsymbol{p}} F_{\boldsymbol{q}}\left(\boldsymbol{x}_{\neq \boldsymbol{p}}, \xi\right)\right| d \xi \leq K_{1}$, and

(iii) $\sup \left\{\operatorname{Var}\left(F_{t}^{*} \nu\right): 0 \leq t \leq 1, \nu \in \mathcal{B}(\Omega), \operatorname{Var} \nu \leq 1\right\} \leq K_{2} ; F_{t}=(t \tilde{F}+(1-t) F)$. Then, for each $\nu \in \mathcal{B}$,

$$
\left|\tilde{F}^{*} \nu-F^{*} \nu\right| \leq K_{2}\left(K_{0}+K_{1}\right) \operatorname{Var} \nu .
$$

b) For use in Section 5 we provide a variant of the above estimate: if assumptions

(i) and (ii) are replaced by

(iv) $\sum_{q \in \Lambda} \sup _{x}\left|\tilde{F}_{q}(\boldsymbol{x})-F_{q}(\boldsymbol{x})\right|^{\frac{1}{2}} \leq K_{3}$ for some $K_{3}>0$,

\footnotetext{
${ }^{14} F: \Omega \rightarrow \Omega$ is a "Lipschitz map", if all $F_{q}(x)$ are Lipschitz with respect to each coordinate $x_{p}$ with uniformly bounded Lipschitz constants. This means in particular that all partial derivatives of all $F_{q}$ exist Lebesgue-a.e., are uniformly bounded and that $F_{q}\left(x+s e_{p}\right)-F_{q}(x)=\int_{0}^{s} \partial_{p} F_{q}\left(x+\xi e_{p}\right) d \xi$.
} 
then, for each $\nu \in \mathcal{B}$,

$$
\left|\tilde{F}^{*} \nu-F^{*} \nu\right| \leq 2 K_{2}^{\frac{1}{2}} K_{3} \operatorname{Var} \nu .
$$

As in [26. Lemma 8] one shows that the assumptions of part 国) of this lemma are satisfied for $F=\Phi_{\epsilon}$ and $\tilde{F}=\Phi_{\epsilon}^{p}$. Hence one can compute

$$
\left|\Phi_{\epsilon}^{*} \mu-\left(\Phi_{\epsilon}^{p}\right)^{*} \mu\right| \leq|\epsilon|\left(8 a_{1}+2 a_{2}+4\right) \operatorname{Var} \mu
$$

provided $|\epsilon|<\min \left\{\frac{1}{6 a_{1}}, \frac{2}{9 a_{2}}\right\}$. Using Lemma 2.2 it is then straightforward to show (compare the proof of [26, Theorem 6])

$$
\left|T_{\epsilon}^{* m_{2}} \mu-T_{\epsilon, p}^{* m_{2}} \mu\right| \leq C m_{2}|\epsilon| \operatorname{Var} \mu .
$$

We are finally at the punch-line: let $h$ be the invariant probability density of the single site map $\tau$. As $\psi(x):=\int_{0}^{1} h(\xi) \varphi\left(\left(T_{\epsilon, p}^{m_{2}} x\right)_{\neq \boldsymbol{p}}, \tau^{m_{2}} \xi\right) d \xi$ does not depend on $x_{\boldsymbol{p}}$, we have $\mu_{\boldsymbol{p}}(\psi)=0$, so that

$$
\begin{aligned}
T_{\epsilon, \boldsymbol{p}}^{* m_{2}} \mu_{\boldsymbol{p}}(\varphi) & =\mu_{\boldsymbol{p}}\left(\varphi \circ T_{\epsilon, \boldsymbol{p}}^{m_{2}}\right)=\mu_{\boldsymbol{p}}\left(\frac{d}{d x_{\boldsymbol{p}}} \int_{0}^{1}\left(\chi_{\left[0, x_{p}\right]}(\xi)-x_{\boldsymbol{p}} h(\xi)\right) \varphi\left(\left(T_{\epsilon, \boldsymbol{p}}^{m_{2}} x\right)_{\neq \boldsymbol{p}}, \tau^{m_{2}} \xi\right) d \xi\right) \\
& =\mu_{\boldsymbol{p}}\left(\frac{d}{d x_{\boldsymbol{p}}} \int_{0}^{1} \mathcal{L}^{m_{2}}\left(\chi_{\left[0, x_{p}\right]}-x_{\boldsymbol{p}} h\right)(\xi) \varphi\left(\left(T_{\epsilon, \boldsymbol{p}}^{m_{2}} x\right)_{\neq \boldsymbol{p}}, \xi\right) d \xi\right)
\end{aligned}
$$

where $\mathcal{L}$ is the transfer operator of the single site map. This means that, calling $\sigma_{0}$ the mixing rate for the single site map,

$$
\left|T_{\epsilon, \boldsymbol{p}}^{* m_{2}} \mu_{\boldsymbol{p}}\right| \leq C \sigma_{0}^{m_{2}} \operatorname{Var} \mu_{\boldsymbol{p}} .
$$

Combining this equation with (3.10) and (3.17) yields

$$
\operatorname{Var}\left(T_{\epsilon}^{* m} \mu_{\boldsymbol{p}}\right) \leq a^{\prime} \lambda^{-m_{1}} \operatorname{Var} \mu_{\boldsymbol{p}}+b C m_{2}|\epsilon| \operatorname{Var} \mu_{\boldsymbol{p}}+b C \sigma_{0}^{m_{2}} \operatorname{Var} \mu_{\boldsymbol{p}} .
$$

Setting $\sigma_{1}:=\max \left\{\lambda^{-1}, \sigma_{0}\right\}^{\frac{1}{4}}<1$ and $m_{1}=m_{2}$, there is, for $m$ large enough, $\epsilon(m)>0$ such that for $|\epsilon|<\epsilon(m)$ holds

$$
\operatorname{Var}\left(T_{\epsilon}^{* m} \mu_{\boldsymbol{p}}\right) \leq \sigma_{1}^{m} \operatorname{Var} \mu_{\boldsymbol{p}} .
$$

In view of Lemma 3.1 we conclude that, for each $\bar{\mu} \in \overline{\mathcal{B}}_{\Lambda}$,

$$
\left\|\bar{T}_{\epsilon, m} \bar{\mu}\right\| \leq C(m w)^{d} \sigma_{1}^{m}\|\bar{\mu}\| .
$$

At this point we can choose $m$ large enough so that $\left[C(m w)^{d}\right]^{\frac{1}{m}} \sigma_{1}=: \sigma<1$, whereby obtaining

$$
\left\|\bar{T}_{\epsilon, m} \bar{\mu}\right\| \leq \sigma^{m}\|\bar{\mu}\| .
$$

We now conclude the argument by using equation (3.2) and (3.9)

$$
\left\|T_{\epsilon}^{* p m} \mu\right\|_{\theta}=\left\|P \bar{T}_{\epsilon, m}^{p} \Psi(\mu)\right\|_{\theta} \leq 2 \min \left\{|\Lambda|,|e \ln \theta|^{-1}\right\} \sigma^{p m} \operatorname{Var} \mu .
$$

By the usual trick of writing $n=p m+q, q<m$ and Lemma 2.2 we finally have

$$
\left\|T_{\epsilon}^{* n} \mu\right\|_{\theta} \leq C \min \left\{|\Lambda|,|e \ln \theta|^{-1}\right\} \sigma^{n} \operatorname{Var} \mu,
$$

for each $\mu \in \mathcal{B}^{0}$ and $\theta \in(0,1)$. This finishes the proof of Lemma 2.3 


\section{Proof of Theorem 2.1}

Having obtained the exponential estimate (3.20), the assertions of Theorem 2.1 can be proved along well known lines. For our convenience we follow once more 26].

The existence of a $T_{\epsilon}$-invariant probability measure $\mu_{\epsilon} \in \mathcal{B}$ follows from a weak compactness argument as in [26. Theorem 4]. The uniqueness of such a measure $\mu_{\epsilon}$ in $\mathcal{B}$ is an immediate consequence of (3.20).

Uniqueness in $\mathcal{M}_{v}(\Omega)$ follows by a standard approximation argument. Assume there exists an invariant measure $\tilde{\mu} \in \mathcal{M}_{v}(\Omega)$. By definition, given $\delta, \eta>0, \tilde{\mu}$ can be approximated by a measure $\mu_{\delta, \eta}$ such that $\left|\tilde{\mu}-\mu_{\delta, \eta}\right| \leq \delta$ and $\operatorname{Var}_{\Lambda^{\prime}} \mu_{\delta} \leq C_{\delta, \eta} e^{\eta\left|\Lambda^{\prime}\right|^{\frac{1}{d}}}$ for any $\Lambda^{\prime} \in \mathcal{I}$.

Let $\varphi$ be a function depending only on the variables belonging to a box $\Lambda_{0} \in \mathcal{I}$, so that $\varphi \circ T_{\epsilon}^{n}$ depends only on the variables in the $n w$-neighborhood $\Lambda_{n}$ of $\Lambda_{0}$. Then

$$
\tilde{\mu}(\varphi)=T_{\epsilon}^{* n}\left(\tilde{\mu}-\mu_{\delta, \eta}\right)(\varphi)+T_{\epsilon}^{* n}\left(\mu_{\delta, \eta}-\mu_{\epsilon}\right)(\varphi)+\mu_{\epsilon}(\varphi)
$$

We must prove that $\tilde{\mu}(\varphi)=\mu_{\epsilon}(\varphi)$. As $\left|T_{\epsilon}^{* n}\left(\tilde{\mu}-\mu_{\delta, \eta}\right)\right| \leq\left|\tilde{\mu}-\mu_{\delta, \eta}\right| \leq \delta$ with an arbitrary $\delta>0$, it just remains to show that the second term can be made as small as we like by choosing $\eta$ and $n$ appropriate.

Given a measure $\mu$ let $\mu_{\Lambda_{n}}$ be its marginal with respect to the box $\Lambda_{n}$. Given a measure $\mu$ on $I^{\Lambda_{n}}$ it is convenient to extend it to a measure $\mu^{\prime}$ on all $I^{\Lambda}$ by simply tensoring it with the Lebesgue measure on the complement of $\Lambda_{n}$; note that such an extension does not increase the bounded variation of the measure. With these conventions,

$$
T_{\epsilon}^{* n}\left(\mu_{\delta, \eta}-\mu_{\epsilon}\right)(\varphi)=T_{\epsilon}^{* n}\left(\left[\mu_{\delta, \eta, \Lambda_{n}}\right]^{\prime}-\left[\mu_{\epsilon, \Lambda_{n}}\right]^{\prime}\right)(\varphi)
$$

Hence, by (3.20),

$$
\left|T_{\epsilon}^{* n}\left(\mu_{\delta, \eta}-\mu_{\epsilon}\right)(\varphi)\right| \leq C_{\theta} \theta^{-\left|\Lambda_{0}\right|} \sigma^{n}\left(1+C_{\delta, \eta} e^{\eta\left|\Lambda_{n}\right|^{\frac{1}{d}}}\right)
$$

As $\left|\Lambda_{n}\right| \leq\left|\Lambda_{0}\right|+\left(\left|\Lambda_{0}\right|^{\frac{1}{d}}+2 n w\right)^{d}$ it follows that, for $2 w \eta<|\ln \sigma|$, one can make this term arbitrarily small by choosing $n$ large. Hence $\tilde{\mu}=\mu_{\epsilon}$.

Since in the case $|\Lambda|<\infty$ one has $\mathcal{M}_{v}(\Omega)=\mathcal{M}(\Omega)$, it follows that finite systems have only one measure absolutely continuous with respect to Lebesgue.

If $|\Lambda|=\infty$ and $\Lambda^{\prime} \in \mathcal{I}$, let $\Phi_{\epsilon, \Lambda^{\prime}}\left(x_{\in \Lambda^{\prime}}\right):=\Phi_{\epsilon}\left(x_{\in \Lambda^{\prime}}, 0_{\notin \Lambda^{\prime}}\right)$. Then $\Phi_{\epsilon, \Lambda^{\prime}}$ is still an $\left(a_{1}, a_{2}\right)$-coupling with finite range $w$ for the configuration space $I^{\Lambda^{\prime}}$ and $T_{\epsilon, \Lambda^{\prime}}:=$ $\Phi_{\epsilon, \Lambda^{\prime}} \circ T_{0}$ gives a dynamics to which all our results apply. Calling $\mu_{\epsilon}^{\Lambda^{\prime}}$ its unique invariant measure absolutely continuous to Lebesgue one can prove, with essentially the same argument as before that, for each $\varphi \in \mathcal{C}^{0}(\Omega)$,

$$
\lim _{\Lambda^{\prime} \rightarrow \Lambda}\left|\mu_{\epsilon}(\varphi)-\left[\mu_{\epsilon}^{\Lambda^{\prime}}\right]^{\prime}(\varphi)\right|=0 .
$$

The exponential mixing in space and time can be obtained exactly as in 26].

Finally, our use of the term $S R B$ for the measure $\mu_{\epsilon}$ is justified by the fact that $\mu$ enjoys the law of large number with respect to a vast class of initial measures related to Lebesgue and, in addition, is stable under smooth random perturbations, that is the random perturbations have a unique invariant measure that converges to $\mu_{\epsilon}$, see [26].

Normally, for finite systems, the criteria for defining the SRB measure are three (e.g., see [36]), the absolutely continuity of the measure along the unstable manifolds, the law of large numbers with respect to Lebesgue for smooth observables, 
the stability with respect to random perturbations. In the infinite case the situation is a bit more subtle since measures tend to be not absolutely continuous one with respect to the other and, in our case, [15] shows that a lot of invariant measures can have marginals absolutely continuous with respect to Lebesgue. Yet, we have shown that, if some moderate regularity is required, then only one invariant measure with absolutely continuous marginals exists, moreover this is the limit obtained by truncating the system to a finite size as (4.4) shows. This together with the fulfillment of the other two requirements is, in our opinion, sufficient to attribute to $\mu_{\epsilon}$ the qualification of SRB.

\section{SHORT RANGE}

Since now the range is infinite it is necessary to decompose the interaction according to space scales, the point being that the interaction on larger scales is smaller and smaller in the weak norm but no control is available on its variation, hence it is necessary to wait longer and longer times for the dynamics to act effectively on it. This forces a more complex book keeping mechanism which is reflected in the necessity of a larger covering Banach space that, with a slight abuse of notation, we will still call $\overline{\mathcal{B}}_{\Lambda}$.

Let $S$ be a positive integer to be fixed later. We define the Banach spaces

$\overline{\mathcal{B}}_{\Lambda}:=\left\{\bar{\mu}:=\left(\mu_{\boldsymbol{q}, t, l}\right): \boldsymbol{q} \in \Lambda ; t \in \mathbb{N}_{S}:=\mathbb{N} \backslash\{1, \ldots, S\} ; l \in\{0, \ldots, t\}, \mu_{\boldsymbol{q}, t, l} \in \mathcal{B}_{\boldsymbol{q}}\right\} ;$ together with the norm

$$
\|\bar{\mu}\|:=\sup _{\boldsymbol{q} \in \Lambda} \sup _{\substack{t \in \mathbb{N}_{S} \\ l \in\{0, \ldots, t\}}}\left(\rho^{t} \alpha^{l} \operatorname{Var} \mu_{\boldsymbol{q}, t, l}+\rho^{-t} \alpha^{l-t}\left|\mu_{\boldsymbol{q}, t, l}\right|\right),
$$

for some constants $\alpha, \rho \in(0,1)$ to be fixed later. Pictorially, one can imagine the above space as a collection of towers at each site $\boldsymbol{q} \in \Lambda$, where the tower $t$ has hight $t$ and the the index $l$ denotes the $l$-th floor in this tower. We can now define the lifted linear dynamics

$$
(\bar{T} \bar{\mu})_{\boldsymbol{q}, t, l}:= \begin{cases}\mu_{\boldsymbol{q}, t, l-1} & \text { if } l>0 \\ \sum_{\{(\boldsymbol{p}, s): \tau(\boldsymbol{q}-\boldsymbol{p}, s)=t\}} \Pi_{\boldsymbol{p}, \boldsymbol{q}}^{*} T_{\epsilon}^{* m(s+1)} \mu_{\boldsymbol{p}, s, s} & \text { if } l=0\end{cases}
$$

where

$$
\tau(\boldsymbol{q}-\boldsymbol{p}, s):= \begin{cases}0 & \text { if }|\boldsymbol{q}-\boldsymbol{p}| \leq s^{2}+S \\ |\boldsymbol{q}-\boldsymbol{p}| & \text { if }|\boldsymbol{q}-\boldsymbol{p}|>s^{2}+S\end{cases}
$$

Roughly speaking, within each tower $s$ at site $\boldsymbol{p}$ the operator $\bar{T}$ pushes each measure one floor up, except for the measure at the top level, which is first transformed according to the dynamics of the whole tower and then distributed (by means of the telescoping operators $\Pi_{\boldsymbol{p}, \boldsymbol{q}}^{*}$ ) to the ground levels of towers at sites $\boldsymbol{q}$ in the following way: If $\boldsymbol{q}$ is close to $\boldsymbol{p}$ (in the sense $|\boldsymbol{q}-\boldsymbol{p}| \leq s^{2}+S$ ) the corresponding measure is mapped to the tower of height $t=0$, whereas if $\boldsymbol{q}$ is farther away from $\boldsymbol{p}$, it is mapped to the tower of height $|\boldsymbol{q}-\boldsymbol{p}|$.

To relate the dynamics of the linear system $\left(\bar{T}, \overline{\mathcal{B}}_{\Lambda}\right)$ with that of the operator $T_{\epsilon}^{* m}$ (for an integer $m$ to be fixed later) we introduce the space $\mathcal{B}_{w}(\theta)$ as the completion of $\mathcal{B}$ with respect to the weak norm

$$
|\mu|_{\theta}:=\sup _{\Lambda_{1} \in \mathcal{I}|\varphi|_{\mathcal{C}^{0}\left(I^{\left.\Lambda_{1}\right)}\right.} \leq 1} \sup ^{\left|\Lambda_{1}\right|}|\mu(\varphi)|
$$


Then we define

$$
\Psi: \mathcal{B}^{0} \rightarrow \overline{\mathcal{B}}_{\Lambda}, \quad(\Psi \mu)_{\boldsymbol{q}, t, l}= \begin{cases}\Pi_{\mathbf{0}, q}^{*} \mu & \text { if } t=l=0 \\ 0 & \text { otherwise }\end{cases}
$$

and

$$
P: \overline{\mathcal{B}}_{\Lambda} \rightarrow \mathcal{B}_{w}(\theta), \quad P \bar{\mu}=\sum_{\boldsymbol{q}, t, l} T_{\epsilon}^{* m l} \mu_{\boldsymbol{q}, t, l} .
$$

It is easy to check that $P \bar{T}^{n}=T_{\epsilon}^{* m n} P$, and hence $P \bar{T}^{n} \Psi=T_{\epsilon}^{* m n}$, for each $n \in \mathbb{N}$, so that the linear system $\left(\bar{T}, \overline{\mathcal{B}}_{\Lambda}\right)$ is indeed an extension of $T_{\epsilon}^{* m}: \mathcal{B}^{0} \rightarrow \mathcal{B}_{w}(\theta)[15$

The following lemma is the main result of this section.

Lemma 5.1. If $\tau$ is Lipschitz and $\Phi_{\epsilon}$ is a short range coupling (see Section 0 for this terminology), then there exist $\sigma \in(0,1)$ and $C, \epsilon_{2}>0$ such that, for all $|\epsilon|<\epsilon_{2}$, $\mu \in \mathcal{B}^{0}(\Omega), \theta \in(0,1)$, and $n \in \mathbb{N}$ holds true

$$
\left|T_{\epsilon}^{* n} \mu\right|_{\theta} \leq C \sigma^{n} \min \left\{|\Lambda|,|e \ln \theta|^{-1}\right\} \operatorname{Var} \mu .
$$

Proof. As in the case of a finite coupling range, estimate (5.3) follows from the fact that $\bar{T}$ is a strict contraction on $\overline{\mathcal{B}}_{\Lambda}$. Namely, we will show that there are $m \in \mathbb{N}$, $\sigma \in(0,1)$, and $\epsilon_{2}>0$ such that for all $\bar{\mu} \in \overline{\mathcal{B}}_{\Lambda}$ holds

$$
\|\bar{T} \bar{\mu}\|=\sup _{\boldsymbol{q}, t, l}\left|(\bar{T} \bar{\mu})_{\boldsymbol{q}, t, l}\right|_{t, l} \leq \sigma^{m}\|\bar{\mu}\|
$$

where $|\mu|_{t, l}:=\rho^{t} \alpha^{l} \operatorname{Var} \mu+\rho^{-t} \alpha^{l-t}|\mu|$, see (5.1).

The case $l \neq 0$ is easy: for all $\boldsymbol{q}$ and $t$ we have

$$
\left|(\bar{T} \bar{\mu})_{q, t, l}\right|_{t, l}=\left|\mu_{q, t, l-1}\right|_{t, l}=\alpha\left|\mu_{q, t, l-1}\right|_{t, l-1} \leq \alpha\|\bar{\mu}\| \leq \sigma^{m}\|\bar{\mu}\|,
$$

where $m>0$ and $\sigma \in\left(\alpha^{\frac{1}{m}}, 1\right)$ will be determined in the course of the proof.

So we assume from now on that $l=0$ and start with the case $t=0 .(\bar{T} \bar{\mu})_{q, 0,0}$ is given by the sum in (5.2) that ranges over indices $\boldsymbol{p}$ and $s$. We begin with the contributions for $s=0$. Without loss of generality we may assume that $m$ is even. By $C$ we denote any constant that may depend on the "ingredients" of the system (like $a, b, \lambda$, etc.) but which is independent of any constant that is to be fixed during the proof (i.e. $S, \alpha, \rho, m, \sigma, \epsilon_{1}$. A crucial choice will be $m=\delta S$ for some $\delta>0$ to be fixed later.) Then

$$
\begin{aligned}
& \left|\Pi_{\boldsymbol{p}, \boldsymbol{q}}^{*} T_{\epsilon}^{* m} \mu_{\boldsymbol{p}, 0,0}\right|_{0,0} \\
& =\operatorname{Var}\left(\Pi_{\boldsymbol{p}, \boldsymbol{q}}^{*} T_{\epsilon}^{* m} \mu_{\boldsymbol{p}, 0,0}\right)+\left|\Pi_{\boldsymbol{p}, \boldsymbol{q}}^{*} T_{\epsilon}^{* m} \mu_{\boldsymbol{p}, 0,0}\right| \\
& \leq 2 \operatorname{Var}\left(T_{\epsilon}^{* m} \mu_{\boldsymbol{p}, 0,0}\right)+2\left|T_{\epsilon}^{* m} \mu_{\boldsymbol{p}, 0,0}\right| \\
& \leq 2 a \lambda^{-\frac{m}{2}} \operatorname{Var}\left(T_{\epsilon}^{* \frac{m}{2}} \mu_{\boldsymbol{p}, 0,0}\right)+2(b+1)\left|T_{\epsilon}^{* \frac{m}{2}} \mu_{\boldsymbol{p}, 0,0}\right| \\
& \leq C \lambda^{-\frac{m}{2}} \operatorname{Var}\left(\mu_{p, 0,0}\right)+C|\epsilon| m \operatorname{Var}\left(\mu_{p, 0,0}\right)+C \sigma_{0}^{\frac{m}{2}} \operatorname{Var}\left(\mu_{p, 0,0}\right) \\
& \leq \sigma_{1}^{m} \operatorname{Var}\left(\mu_{\boldsymbol{p}, 0,0}\right) \\
& \leq \sigma_{1}^{m}\|\bar{\mu}\| \quad\left(\sigma_{1}:=\max \left\{\lambda^{-1}, \sigma_{0}\right\}^{\frac{1}{4}}\right)
\end{aligned}
$$

\footnotetext{
${ }^{15}$ Observe that, since $\sum_{s \in \mathbb{N}_{S}, 0 \leq l \leq s} \rho^{-s} \alpha^{-l}$ does not converge, it is not true that $P \bar{\mu} \in \mathcal{B}(\theta)$ for each $\bar{\mu} \in \overline{\mathcal{B}}_{\Lambda}$, while $\|P\|_{\overline{\mathcal{B}}_{\Lambda} \rightarrow \mathcal{B}_{w}(\theta)} \leq(1-\rho)^{-1}(1-\alpha)^{-1}|e \ln \theta|^{-1}$.
} 
for sufficiently large $m$ and $|\epsilon|<\epsilon(m)$, where we used essentially the same arguments that lead already to (3.18). Summing this over all $\boldsymbol{p}$ for which $\tau(\boldsymbol{q}-\boldsymbol{p}, 0)=t=0$ means that one has to sum over all $\boldsymbol{p}$ for which $|\boldsymbol{q}-\boldsymbol{p}| \leq S$ :

$$
\left|\sum_{\{p: \tau(q-p, 0)=0\}} \Pi_{p, q}^{*} T_{\epsilon}^{* m} \mu_{p, 0,0}\right|_{0,0} \leq C S^{d} \sigma_{1}^{m}\|\bar{\mu}\| \leq \frac{\sigma^{m}}{2}\|\bar{\mu}\|
$$

for a suitable $\sigma \in\left(\sigma_{1}, 1\right)$, provided $m=\delta S$ is sufficiently large.

Next we estimate the contributions for $s \neq 0$ to the sum in (5.2) when $l=0$ and $t=0$. In this case

$$
\begin{aligned}
\left|\Pi_{\boldsymbol{p}, \boldsymbol{q}}^{*} T_{\epsilon}^{* m(s+1)} \mu_{\boldsymbol{p}, s, s}\right|_{0,0} & =\operatorname{Var}\left(\Pi_{\boldsymbol{p}, \boldsymbol{q}}^{*} T^{* m(s+1)} \mu_{\boldsymbol{p}, s, s}\right)+\left|\Pi_{\boldsymbol{p}, \boldsymbol{q}}^{*} T^{* m(s+1)} \mu_{\boldsymbol{p}, s, s}\right| \\
& \leq 2 \operatorname{Var}\left(T^{* m(s+1)} \mu_{\boldsymbol{p}, s, s}\right)+2\left|T^{* m(s+1)} \mu_{\boldsymbol{p}, s, s}\right| \\
& \leq 2 a \lambda^{-m(s+1)} \operatorname{Var}\left(\mu_{p, s, s}\right)+2(1+b)\left|\mu_{p, s, s}\right| \\
& \leq\left[2 a \lambda^{-m(s+1)} \rho^{-s} \alpha^{-s}+2(1+b) \rho^{s}\right]\left|\mu_{p, s, s}\right|_{s, s} .
\end{aligned}
$$

As $s>S$ and $|\boldsymbol{q}-\boldsymbol{p}| \leq s^{2}+S$ in the case under consideration, we conclude

$$
\begin{aligned}
& \left|\sum_{\left\{(\boldsymbol{p}, s): s \in \mathbb{N}_{S} \backslash\{0\}, \tau(\boldsymbol{q}-\boldsymbol{p}, s)=0\right\}} \Pi_{p, q}^{*} T_{\epsilon}^{* m(s+1)} \mu_{\boldsymbol{p}, s, s}\right|_{0,0} \\
& \leq C \sum_{s=S+1}^{\infty}\left(s^{2}+S\right)^{d}\left[2 a \lambda^{-m(s+1)} \rho^{-s} \alpha^{-s}+2(1+b) \rho^{s}\right]\|\bar{\mu}\| \\
& \leq C \sigma_{2}^{S}\|\bar{\mu}\| \leq \frac{\sigma^{m}}{2}\|\bar{\mu}\|
\end{aligned}
$$

for suitable $\sigma_{2} \in(\rho, 1)$ and $\sigma \in\left(\sigma_{2}^{\delta / 2}, 1\right)$, provided $\lambda^{-m}<\alpha \rho^{2}$ and $m=\delta S$ is sufficiently large.

We finally turn to the case $l=0$ and $t \neq 0$. For this we will need the following estimate: There are $\beta \in(0,1)$ and $\delta>0$ such that

$$
\left|\Pi_{p, q}^{*} T_{\epsilon}^{* m(s+1)} \mu_{p, s, s}\right| \leq C_{m} \beta^{|q-p|} \operatorname{Var}\left(\mu_{p, s, s}\right)
$$

provided $m(s+1)<\delta|\boldsymbol{q}-\boldsymbol{p}|$. The proof will be given below.

Now, since $t \neq 0$, the condition $\tau(\boldsymbol{q}-\boldsymbol{p}, s)=t$ in the summation in (5.2) means that $t=|\boldsymbol{q}-\boldsymbol{p}|>s^{2}+S$. In particular, as $m=\delta S$, we have $m(s+1)=\delta S(s+1)<$ $\delta\left(s^{2}+S\right)<\delta|\boldsymbol{q}-\boldsymbol{p}|$ so that (5.8) is applicable. Therefore

$$
\begin{aligned}
& \left|\Pi_{p, q}^{*} T_{\epsilon}^{* m} \mu_{p, s, s}\right|_{t, 0} \\
& =\rho^{t} \operatorname{Var}\left(\Pi_{p, q}^{*} T_{\epsilon}^{* m(s+1)} \mu_{p, s, s}\right)+\rho^{-t} \alpha^{-t}\left|\Pi_{p, q}^{*} T_{\epsilon}^{* m(s+1)} \mu_{p, s, s}\right| \\
& \leq 2 a \rho^{t} \lambda^{-m(s+1)} \operatorname{Var}\left(\mu_{p, s, s}\right)+2 b \rho^{t}\left|\mu_{p, s, s}\right|+\rho^{-t} \alpha^{-t} C_{m} \beta^{|q-p|} \operatorname{Var}\left(\mu_{p, s, s}\right) \\
& \leq\left(2 a \rho^{t}\left(\rho \alpha \lambda^{m}\right)^{-s}+2 b \rho^{s} \rho^{t}+(\rho \alpha)^{-s-t} C_{m} \beta^{|q-p|}\right)\left|\mu_{p, s, s}\right|_{s, s} .
\end{aligned}
$$

Hence, observing that $t=|\boldsymbol{q}-\boldsymbol{p}|>S$ and $s<\sqrt{t}$,

$$
\begin{aligned}
& \left|\sum_{\{(\boldsymbol{p}, s): \tau(\boldsymbol{q}-\boldsymbol{p}, s)=t\}} \Pi_{\boldsymbol{p}, \boldsymbol{q}}^{*} T_{\epsilon}^{* m} \mu_{\boldsymbol{p}, s, s}\right|_{t, 0} \\
& \leq \sum_{s=0}^{\sqrt{t}} C t^{d}\left(\rho^{t}\left(\rho \alpha \lambda^{m}\right)^{-s}+\rho^{s} \rho^{t}+(\rho \alpha)^{-s-t} C_{m} \beta^{t}\right)\|\bar{\mu}\| \\
& \leq C \sigma_{3}^{S}\|\bar{\mu}\| \leq \sigma^{m}\|\bar{\mu}\|,
\end{aligned}
$$


for suitable $\sigma_{3} \in(\rho, 1)$ and $\sigma \in\left(\sigma_{3}^{\frac{1}{2 \delta}}, 1\right)$, provided $\alpha \rho^{2}>\beta$ and $S=\delta^{-1} m$ is sufficiently large.

Putting together (5.5), (5.6), (5.7), and (5.9) yields

$$
\|\bar{T} \bar{\mu}\| \leq \sigma^{m}\|\bar{\mu}\|
$$

for some $m>0$ and $\sigma \in(0,1)$. This concludes the proof of Lemma 5.1

Proof of estimate (5.8). The basic idea of the proof is to approximate $T_{\epsilon}^{m(s+1)}$ by a map $\tilde{T}_{s}=\tilde{T}_{\epsilon, \boldsymbol{p}, \boldsymbol{q}, m(s+1)}$ with the property that $\partial_{\boldsymbol{p}}\left(\left(\tilde{T}_{s}\right)_{\boldsymbol{q}^{\prime}}\right)=0$ if $\left|\boldsymbol{q}^{\prime}-\boldsymbol{p}\right| \geq|\boldsymbol{q}-\boldsymbol{p}|$. To this end recall the map $\bar{\iota}_{p}: I^{\Lambda} \rightarrow I^{\Lambda}$ from Section 3 $\left(\bar{\iota}_{p}(\boldsymbol{x})\right)_{q^{\prime}}=x_{\boldsymbol{q}^{\prime}}$ if $\boldsymbol{q}^{\prime} \neq \boldsymbol{p}$ and $\left(\bar{\iota}_{\boldsymbol{p}}(\boldsymbol{x})\right)_{q^{\prime}}=0$ if $\boldsymbol{q}^{\prime}=\boldsymbol{p}$. Then define

$$
\left(\tilde{T}_{s}(\boldsymbol{x})\right)_{\boldsymbol{q}^{\prime}}= \begin{cases}\left(T_{\epsilon}^{m(s+1)}(\boldsymbol{x})\right)_{\boldsymbol{q}^{\prime}} & \text { if }\left|\boldsymbol{q}^{\prime}-\boldsymbol{p}\right|<c^{-2}|\boldsymbol{q}-\boldsymbol{p}| \\ \left(T_{\epsilon}^{m(s+1)}\left(\bar{\iota}_{\boldsymbol{p}}(\boldsymbol{x})\right)_{\boldsymbol{q}^{\prime}}\right. & \text { if }\left|\boldsymbol{q}^{\prime}-\boldsymbol{p}\right| \geq c^{-2}|\boldsymbol{q}-\boldsymbol{p}|\end{cases}
$$

Note first that $\left(\Pi_{p, q} \varphi\right)\left(\tilde{T}_{s}(\boldsymbol{x})\right)$ is constant as a function of $x_{\boldsymbol{p}}$ because $c \geq 1$. It follows that

$$
\Pi_{p, q}^{*} \tilde{T}_{s}^{*} \mu=0 \quad \text { if } \mu \in \mathcal{B}_{p}
$$

Hence, recalling that $\mu_{p, s, s} \in \mathcal{B}_{p}$, we see that

$$
\left|\Pi_{\boldsymbol{p}, \boldsymbol{q}}^{*} T_{\epsilon}^{* m(s+1)} \mu_{\boldsymbol{p}, s, s}\right|=\left|\Pi_{\boldsymbol{p}, \boldsymbol{q}}^{*}\left(T_{\epsilon}^{* m(s+1)}-\tilde{T}_{s}^{*}\right) \mu_{\boldsymbol{p}, s, s}\right| \leq 2\left|\left(T_{\epsilon}^{* m(s+1)}-\tilde{T}_{s}^{*}\right) \mu_{\boldsymbol{p}, s, s}\right| .
$$

The latter quantity can be bounded using Lemma 3.2b To this end let us check the hypotheses of that lemma.

$$
\left|\left(\tilde{T}_{s}(\boldsymbol{x})-T_{\epsilon}^{m(s+1)}(\boldsymbol{x})\right)_{\boldsymbol{q}^{\prime}}\right| \leq \begin{cases}0 & \text { if }\left|\boldsymbol{q}^{\prime}-\boldsymbol{p}\right|<c^{-2}|\boldsymbol{q}-\boldsymbol{p}| \\ \left|\left(D T_{\epsilon}^{m(s+1)}\right)_{\boldsymbol{q}^{\prime} \boldsymbol{p}}\right|_{\infty} & \text { if }\left|\boldsymbol{q}^{\prime}-\boldsymbol{p}\right| \geq c^{-2}|\boldsymbol{q}-\boldsymbol{p}|\end{cases}
$$

To estimate the derivative notice that $0 \leq\left|\left(D \Phi_{\epsilon}\right)_{q^{\prime} p}\right| \leq \delta_{q^{\prime} p}+2 L|\epsilon| \gamma^{\left|q^{\prime}-p\right|}$ so that $0 \leq\left|\left(D \Phi_{\epsilon}\right)_{q^{\prime} p}\right|^{\frac{1}{2}} \leq \delta_{q^{\prime} p}+\sqrt{2 L|\epsilon|} \gamma^{\frac{1}{2}\left|q^{\prime}-p\right|}=:(\mathbf{I d}+\sqrt{2 L|\epsilon|} B)_{q^{\prime} p}$. Hence, setting $\lambda_{+}:=\left|\tau^{\prime}\right|_{\infty}$, by the triangular inequality

$$
\left|\left(D T_{\epsilon}^{n}\right)_{q^{\prime} p}\right| \leq \lambda_{+}^{n}\left\{\left([\mathbf{I d}+\sqrt{2 L|\epsilon|} B]^{n}\right)_{q^{\prime} p}\right\}^{2} .
$$

Using a Cramér type estimate as in [27] this leads to the following bound for $K_{3}$ : let $n=m(s+1)$ and $r=|\boldsymbol{q}-\boldsymbol{p}|$ so that $n<\delta r$. Then, for any $t>0$,

$$
\begin{aligned}
& \sum_{q^{\prime}}\left|\left(\tilde{T}_{s}(\boldsymbol{x})-T_{\epsilon}^{m(s+1)}(\boldsymbol{x})\right)_{q^{\prime}}\right|^{\frac{1}{2}} \\
& \leq \sum_{\left|\boldsymbol{q}^{\prime}-p\right| \geq c^{-2} r} \lambda_{+}^{\frac{n}{2}}\left([\mathbf{I d}+\sqrt{2 L|\epsilon|} B]^{n}\right)_{\boldsymbol{q}^{\prime} p} \\
& \leq \sum_{\left|q^{\prime}-p\right| \geq c^{-2} r} \lambda_{+}^{\frac{1}{2} \delta r}\left([\mathbf{I d}+\sqrt{2 L|\epsilon|} B]^{\delta r}\right)_{q^{\prime} p} e^{t\left|q^{\prime}-p\right|-t c^{-2} r} \\
& \leq\left(\lambda_{+}^{\frac{\delta}{2}} e^{-t c^{-2}}\right)^{r} \sum_{q^{\prime} \in \mathbb{Z}^{d}}\left([\mathbf{I d}+\sqrt{2 L|\epsilon|} B]^{\delta r}\right)_{q^{\prime} 0} e^{t\left|q^{\prime}\right|} \\
& \leq\left(\lambda_{+}^{\frac{\delta}{2}} \psi(t)^{\delta} e^{-t c^{-2}}\right)^{r}=: \beta^{r}=\beta^{|\boldsymbol{q}-p|}
\end{aligned}
$$

where $\psi(t):=\sum_{q^{\prime} \in \mathbb{Z}^{d}}\left(\delta_{q^{\prime} 0}+\sqrt{2 L|\epsilon|} \gamma^{\frac{1}{2}\left|q^{\prime}\right|}\right) e^{t\left|q^{\prime}\right|}$. Clearly, $|\psi(t)|<\infty$ for $t \in$ $\left(0, \frac{1}{2}|\ln \gamma|\right)$. Hence, if we fix such a $t$ and choose $\delta>0$ sufficiently small, then $\beta \in(0,1)$. (These choices are uniform for $\epsilon$ in a neighbourhood of 0 .) 
The proof that $K_{2}$ can be taken to be some fixed constant (depending on $m$ but not on $\boldsymbol{p}$ and $\boldsymbol{q}$ ) is completely standard and it is left to the reader. Accordingly, Lemma 3.2 yields

$$
\left|\Pi_{p, q}^{*} T_{\epsilon}^{* m(s+1)} \mu_{p, s, s}\right| \leq C_{m} \beta^{|p-q|} \operatorname{Var}\left(\mu_{p, s, s}\right)
$$

and that is (5.8).

Lemma [5.1 and (5.3) are the equivalent of Lemma 2.3] and (3.19) which where the basic ingredients to prove Theorem 2.1 in the finite range case. These results can be used now in a similar way to obtain the corresponding result in the short range case.

Proof of Theorem 2.4. The proof follows the one of Theorem 2.1] let us outline the main points.

The uniqueness of the invariant measure in $\mathcal{B}_{\Lambda}$ follows trivially from Lemma 5.1 On the other hand, the approximation argument is now more subtle since one can no longer use the finite range property in (4.2). Nevertheless, using large deviation type estimates like in the proof of equation (5.8) one can show that (4.2) continues to hold if modulo another small error term.

The same remarks apply to obtaining the spatial decay of correlation out of the temporal ones: again one has to treat explicitly very long range effect by showing that they produce a very small contribution.

Finally, the reasons to call the above invariant measure SRB remain unchanged from the short range case.

\section{Appendix}

Proof of Lemma 3.2 It suffices to estimate $\left(\tilde{F}^{*} \nu-F^{*} \nu\right)(\varphi)$ for a test function $\varphi$ with $|\varphi|_{\mathcal{C}^{0}(\Omega)} \leq 1$ :

$$
\begin{aligned}
& \left(\tilde{F}^{*} \nu-F^{*} \nu\right)(\varphi)=\int_{\Omega} \varphi(\tilde{F} \boldsymbol{x})-\varphi(F \boldsymbol{x}) d \nu(\boldsymbol{x})=\int_{\Omega} \int_{0}^{1} \frac{\partial}{\partial t}\left(\varphi\left(F_{t} \boldsymbol{x}\right)\right) d t d \nu(\boldsymbol{x}) \\
& =\int_{0}^{1} \int_{\Omega} \sum_{\boldsymbol{q} \in \Lambda} \partial_{\boldsymbol{q}} \varphi\left(F_{t} \boldsymbol{x}\right) \frac{\partial}{\partial t} F_{t, \boldsymbol{q}}(\boldsymbol{x}) d \nu(\boldsymbol{x}) d t=\int_{0}^{1} \sum_{\boldsymbol{q} \in \Lambda} F_{t}^{*}\left(\left(\tilde{F}_{\boldsymbol{q}}-F_{\boldsymbol{q}}\right) \cdot \nu\right)\left(\partial_{\boldsymbol{q}} \varphi\right) d t
\end{aligned}
$$

so that

$$
\begin{aligned}
& \left|\tilde{F}^{*} \nu-F^{*} \nu\right| \leq \sum_{\boldsymbol{q} \in \Lambda} K_{2} \operatorname{Var}\left(\left(\tilde{F}_{\boldsymbol{q}}-F_{\boldsymbol{q}}\right) \cdot \nu\right) \\
& \leq K_{2} \sum_{\boldsymbol{q} \in \Lambda}\left(\left|\tilde{F}_{\boldsymbol{q}}-F_{\boldsymbol{q}}\right|_{\infty}+\sup _{\boldsymbol{p} \in \Lambda} \sup _{\boldsymbol{x}_{\neq p}} \int_{I}\left|\partial_{\boldsymbol{p}} \tilde{F}_{\boldsymbol{q}}\left(\boldsymbol{x}_{\neq \boldsymbol{p}}, \xi\right)-\partial_{\boldsymbol{p}} F_{\boldsymbol{q}}\left(\boldsymbol{x}_{\neq \boldsymbol{p}}, \xi\right)\right| d \xi\right) \operatorname{Var} \nu \\
& \leq K_{2}\left(K_{0}+K_{1}\right) \operatorname{Var} \nu .
\end{aligned}
$$

This proves part af of the lemma.

The above estimate is, in some sense, too good for our needs in Section 5 where it may be hard to verify that $K_{1}<\infty$. It is then convenient to have a rougher estimate. To this end let us define the function $R \in \mathcal{C}^{0}\left(\mathbb{R}^{\Lambda}, \Omega\right)$ by

$$
R(\boldsymbol{x})_{\boldsymbol{q}}= \begin{cases}0 & \text { if } x_{\boldsymbol{q}}<0 \\ x_{\boldsymbol{q}} & \text { if } x_{\boldsymbol{q}} \in[0,1] \\ 1 & \text { it } x_{\boldsymbol{q}}>1\end{cases}
$$


and let $\bar{\varphi}:=\varphi \circ R$. Next, define $\kappa, \kappa_{\eta}: \mathbb{R} \rightarrow[0, \infty), \kappa(y):=\max \{1-|y|, 0\}$ and $\kappa_{\eta}(y):=\eta^{-1} \kappa\left(\eta^{-1} y\right)$. For each $\Lambda_{1} \in \mathcal{I}$, we introduce, for each $\bar{\eta}=\left(\eta_{q}\right)_{q \in \Lambda}$, the convolution operators $Q_{\bar{\eta}, \Lambda_{1}}$ on $\mathcal{C}^{0}(\Omega)$ :

$$
\left(Q_{\bar{\eta}, \Lambda_{1}} \varphi\right)(\boldsymbol{x}):=\int_{\mathbb{R}^{\Lambda_{1}}} \prod_{\boldsymbol{p} \in \Lambda_{1}} \kappa_{\eta_{p}}\left(x_{\boldsymbol{p}}-y_{\boldsymbol{p}}\right) \bar{\varphi}\left(\boldsymbol{x}_{\notin \Lambda_{1}}, \boldsymbol{y}\right) d \boldsymbol{y} \nu(d \boldsymbol{x}) .
$$

Not surprisingly, it holds the estimate

$$
\left|Q_{\bar{\eta}, \Lambda_{1}}^{*} \nu-\nu\right| \leq \frac{1}{3} \sum_{q \in \Lambda_{1}} \eta_{q} \operatorname{Var} \nu .
$$

In fact,

$$
\begin{aligned}
& \nu\left(Q_{\bar{\eta}, \Lambda_{1}} \varphi-\varphi\right)=\int_{\Omega} \int_{\mathbb{R}^{\Lambda_{1}}} \prod_{\boldsymbol{p} \in \Lambda_{1}} \kappa_{\eta_{p}}\left(x_{\boldsymbol{p}}-y_{\boldsymbol{p}}\right)\left[\bar{\varphi}\left(\boldsymbol{x}_{\notin \Lambda_{1}}, \boldsymbol{y}\right)-\bar{\varphi}(\boldsymbol{x})\right] d \boldsymbol{y} \nu(d \boldsymbol{x}) \\
& =\int_{0}^{1} d t \int_{\Omega} \int_{\mathbb{R}^{\Lambda_{1}}} \prod_{p \in \Lambda_{1}} \kappa_{\eta_{p}}\left(x_{\boldsymbol{p}}-y_{p}\right) \frac{d}{d t} \bar{\varphi}\left(\boldsymbol{x}_{\notin \Lambda_{1}}, \boldsymbol{x}_{\in \Lambda_{1}}+t\left(\boldsymbol{y}-\boldsymbol{x}_{\in \Lambda_{1}}\right)\right) d \boldsymbol{y} \nu(d \boldsymbol{x}) \\
& =\int_{0}^{1} d t \int_{\Omega} \int_{\mathbb{R}^{\Lambda_{1}}} \prod_{\boldsymbol{p} \in \Lambda_{1}} \kappa_{\eta_{p}}\left(z_{p}\right) \sum_{\boldsymbol{q} \in \Lambda_{1}} \partial_{q} \bar{\varphi}\left(\boldsymbol{x}_{\notin \Lambda_{1}}, \boldsymbol{x}_{\in \Lambda_{1}}+t \boldsymbol{z}\right) z_{\boldsymbol{q}} d \boldsymbol{z} \nu(d \boldsymbol{x}) \\
& =\int_{0}^{1} d t \int_{\mathbb{R}^{\Lambda_{1}}} d \boldsymbol{z} \sum_{\boldsymbol{q} \in \Lambda_{1}} z_{q} \prod_{\boldsymbol{p} \in \Lambda_{1}} \kappa_{\eta_{p}}\left(z_{\boldsymbol{p}}\right) \nu\left(\partial_{q} \bar{\varphi}_{t, \boldsymbol{z}}\right)
\end{aligned}
$$

where $\varphi_{t, z}(\boldsymbol{x}):=\bar{\varphi}\left(\boldsymbol{x}_{\notin \Lambda_{1}}, \boldsymbol{x}_{\in \Lambda_{1}}+t \boldsymbol{z}\right)$. From the above formula estimate (6.1) follows, because $\int_{\mathbb{R}}|z| \kappa_{\eta}(z) d z=\frac{\eta}{3}$.

Accordingly, if $\sum_{q \in \Lambda} \eta_{q}<\infty$ we can define

$$
Q_{\bar{\eta}} \varphi:=\lim _{\Lambda_{1} \rightarrow \Lambda} Q_{\bar{\eta}, \Lambda_{1}} \varphi
$$

Then

$$
\begin{aligned}
\left|F^{*} \nu-\tilde{F}^{*} \nu\right| & \leq \frac{2}{3} K_{2} \sum_{q \in \Lambda} \eta_{q} \operatorname{Var} \nu+\sup _{|\varphi| \leq 1} \nu\left(\left(Q_{\bar{\eta}} \varphi\right) \circ F-\left(Q_{\bar{\eta}} \varphi\right) \circ \tilde{F}\right) \\
& \leq \frac{2}{3} K_{2} \sum_{q} \eta_{q} \operatorname{Var} \nu+|\nu| \sum_{q} \eta_{q}^{-1}\left|F_{q}-\tilde{F}_{q}\right|_{\infty} .
\end{aligned}
$$

Now, for all the $\boldsymbol{q}$ for which $\left|F_{\boldsymbol{q}}-\tilde{F}_{\boldsymbol{q}}\right|_{\infty} \neq 0$, choose $\eta_{\boldsymbol{q}}=K_{2}^{-\frac{1}{2}}\left|F_{\boldsymbol{q}}-\tilde{F}_{\boldsymbol{q}}\right|_{\infty}^{\frac{1}{2}}$ to get

$$
\left|F^{*} \nu-\tilde{F}^{*} \nu\right| \leq 2 K_{2}^{\frac{1}{2}} \operatorname{Var} \nu \sum_{q}\left|F_{q}-\tilde{F}_{q}\right|_{\infty}^{\frac{1}{2}} \leq 2 K_{2}^{\frac{1}{2}} K_{3} \operatorname{Var} \nu
$$

This finishes the proof of part (b) of the lemma.

\section{REFERENCES}

[1] V. Baladi, M. Degli Eposti, S. Isola, E. Järvenpää, A. Kupiainen, The spectrum of weakly coupled map lattices, J. Math. Pures Appl. 77 (1998), 539-584.

[2] V. Baladi, H.H Rugh, Floquet spectrum of weakly coupled map lattices, Commun. Math. Phys. 220 (2001), 561-582.

[3] J.-B. Bardet, Limit theorems for coupled analytic maps, Probab. Th. Rel. Fields 124 (2002), 151-177.

[4] J. Bricmont, A. Kupiainen, Coupled analytic maps, Nonlinearity 8 (1995), no. 3, 379-396.

[5] J. Bricmont, A. Kupiainen, High temperature expansions and dynamical systems, Comm. Math. Phys. 178 (1996), no. 3, 703-732. 
[6] L.A. Bunimovich, Coupled map lattices: one step forward and two steps back, Chaos, order and patterns: aspects of nonlinearity - the "gran finale" (Como, 1993). Phys. D 86 (1995), no. $1-2,248-255$.

[7] L.A. Bunimovich, Ya.G. Sinai, Space-time chaos in coupled map lattices, Nonlinearity 1 (1988), 491-516.

[8] T. Fischer, H.H. Rugh, Transfer operators for coupled analytic maps, Ergod. Th.\& Dynam. Sys. 20 (2000), 109-143.

[9] G. Gielis, R.S. MacKay, Coupled map lattices with phase transition, Nonlinearity 13 (2000), $867-888$

[10] V.M. Gundlach, D.A. Rand, Spatio-temporal chaos: 1. Hyperbolicity, structural stability, spatio-temporal shadowing and symbolic dynamics, Nonlinearity 6 (1991), 165-200.

[11] V.M. Gundlach, D.A. Rand, Spatio-temporal chaos: 2. Unique Gibbs states for higherdimensional symbolic systems, Nonlinearity 6 (1993), 201-214.

[12] V.M. Gundlach, D.A. Rand, Spatio-temporal chaos: 3. Natural spatio-temporal measures for coupled circle map lattices, Nonlinearity 6 (1993), 215-230.

[13] V.M. Gundlach, D.A. Rand, Spatio-temporal chaos (Corrigendum), Nonlinearity 9 (1996), 605-606.

[14] E. Järvenpää, A note on weakly coupled expanding maps on compact manifolds, Annales Academiæ Scientiarum Fennicæ Mathematica 24 (1999), 511-517.

[15] E. Järvenpää, M. Järvenpää, On the definition of SRB-measures for coupled map lattices, Comm. Math. Phys. 220 (2001), no. 1, 1-12.

[16] M. Jiang, Equilibrium states for lattice models of hyperbolic type, Nonlinearity 8 (1995), no. $5,631-659$.

[17] M. Jiang, Equilibrium measures for coupled map lattices: existence, uniqueness and finitedimensional approximations, commun. Math. Phys. 193 (1998), 675-712.

[18] M. Jiang, Sinai-Ruelle-Bowen measures for lattice dynamical systems, J. Statist. Phys. 111 (2003), no. 3-4, 863-902.

[19] M. Jiang, A.E. Mazel, Uniqueness and exponential decay of correlations for some twodimensional spin lattice systems, J. Statist. Phys. 82 (1996), no. 3-4, 797-821.

[20] M. Jiang, Ya. Pesin, Equilibrium measures for coupled map lattices: existence, uniqueness and finite-dimensional approximations, Comm. Math. Phys. 193 (1998), no. 3, 675-711.

[21] G. Keller, Coupled map lattice via transfer operators on functions of bounded variation, Stochastic and spatial structures of dynamical systems (Amsterdam, 1995), 71-80, Konink. Nederl. Akad. Wetensch. Verh. Afd. Natuurk. Eerste Reeks 45 North-Holland, Amsterdam, 1996.

[22] G. Keller, Mixing for finite systems of coupled tent maps, Tr. Mat. Inst. Steklova 216 (1997), Din. Sist. i Smezhnye Vopr., 320-326; translation in Proc. Steklov Inst. Math. 1997, no. 1 (216), 315-32

[23] G. Keller, An ergodic theoretic approach to mean field coupled maps, Fractal geometry and stochastics, II (Greifswald/Koserow, 1998), 183-208, Progr. Probab., 46, Birkhäuser, Basel, 2000.

[24] G. Keller, M.Künzle, Transfer operators for coupled map lattices, Ergodic Theory Dynam. Systems 12 (1992), no. 2, 297-318.

[25] G. Keller, C. Liverani, Coupled map lattices without cluster expansion, Discrete and Continuous Dynamical Systems, 11, n.2,3, 325-335 (2004).

[26] G.Keller, C.Liverani, A spectral gap for a one-dimensional lattice of coupled piecewise expanding interval maps, to appear in Lecture Notes in Physics, Springer.

[27] G. Keller, R. Zweimüller, Unidirectionally coupled interval maps: between dynamics and statistical mechanics, Nonlinearity 15 (2002), no. 1, 1-24.

[28] M. Künzle: Invariante Maße für gekoppelte Abbildungsgitter, Dissertation, Universität Erlangen (1993)

[29] Ch. Maes, A. van Moffaert, Stochastic stability of weakly coupled map lattices, Nonlinearity 10 (1997), 715-730.

[30] J. Miller, D.A. Huse, Macroscopic equilibrium from microscopic irreversibility in a chaotic coupled-map lattice, Pys. Rev. E, 48, 2528-2535 (1993).

[31] Ya.B. Pesin, Ya.G. Sinai, Space-time chaos in chains of weakly interacting hyperbolic mappings, adv.Sov.Math. 3 (1991), 165-198. 
[32] H.H. Rugh, Coupled maps and analytic function spaces, Ann. Sci. École Norm. Sup. (4) 35 (2002), no. 4, 489-535.

[33] M. Schmitt: $B V$-spectral theory for coupled map lattices, Dissertation, Universität Erlangen (2003). See also: Nonlinearity 17, 671-690 (2004).

[34] D.L. Volevich, Kinetics of coupled map lattices, Nonlinearity 4 (1991), 37-45.

[35] D.L. Volevich, Construction of an analogue of Bowen-Sinai measure for a multidimensional lattice of interacting hyperbolic mappings, Russ. Acad. Math. Sbornink 79, 347-363 (1994).

[36] L.-S.Young, What are SRB measures, and which dynamical systems have them, Dedicated to David Ruelle and Yasha Sinai on the occasion of their 65th birthdays. J. Statist. Phys. 108 (2002), no. 5-6, 733-754.

Mathematisches Institut, Universität Erlangen-NÜrnberg, Bismarckstr. 1 1/2, 91054 Erlangen, Germany

E-mail address: keller@mi.uni-erlangen.de

Carlangelo Liverani, Dipartimento di Matematica, il Università di Roma (Tor Vergata), Via della Ricerca Scientifica, 00133 Roma, Italy.

E-mail address: liverani@mat.uniroma2.it 